\title{
RECONCILING DARK MATTER AND SOLAR NEUTRINOS
}

\author{
J. T. Peltoniemi ${ }^{*}$ and D. Tommasini ${ }^{\dagger}$ \\ Instituto de Física Corpuscular - IFIC/C.S.I.C. \\ Dept. de Física Teòrica, Universitat de València \\ 46100 Burjassot, València, SPAIN \\ and
}

\author{
J. W. F. Valle ${ }^{\ddagger}$
}

Theoretical Physics Division, CERN

CH-1211 Geneve 23, Switzerland

\begin{abstract}
We present a simple model for neutrino dark matter in which neutrino masses arise radiatively and the solar neutrino data are explained via the MSW effect. The dark matter scale arises at the one-loop level while the MSW scale arises only in two-loops. The model is compatible with all observational facts and allows observable $\nu_{e} \nu_{\tau}$ or $\nu_{\mu} \nu_{\tau}$ oscillation rates in the laboratory if the limits from primordial big bang nucleosynthesis (BBN) are taken conservatively. In addition, it can be probed by searching for muon number violating processes such as $\mu \rightarrow e+\gamma$, and $\mu \rightarrow 3 e$. These rates can well lie within the sensitivities of present experiments. Finally, if we ignore BBN limits we can have also a common explanation for the atmospheric neutrino deficit via $\nu_{\mu}$ oscillations to a sterile neutrino $\nu_{S}$ with maximal mixing and $10^{-2}-10^{-3} \mathrm{eV}^{2}$.
\end{abstract}

CERN-TH.6624/92

August 1992

${ }^{1}$ Permanent address, Instituto de Física Corpuscular - IFIC/C.S.I.C.

*Bitnet PELTONIE@EVALVX

${ }^{\dagger}$ Bitnet TOMMASIN@EVALUN11

‡Bitnet VALLE@EVALUN11 - Decnet 16444::VALLE 


\section{Introduction}

Two important hints for neutrino masses arising from cosmology and astrophysics are provided by the dark matter (DM) and solar neutrino problems. Recent data on large-scale structure in the Universe suggest the existence of a hot component in the dark matter [1]. If at least one neutrino is massive and stable on cosmological scales, it could provide this hot dark matter, or part of it, provided its mass lies somewhere in the range $1 \mathrm{eV} \lesssim m_{D M} \lesssim 50 \mathrm{eV}$. On the other hand the most natural MSW neutrino oscillation interpretation of the solar neutrino data (including the recent GALLEX data on pp neutrinos $)[2,3,4,5]$ suggests much lower values of neutrino masses: $\delta m_{\odot}^{2} \sim 10^{-5} e V^{2}$.

In principle these two scales $m_{D M}$ and $m_{\odot}$ may be accommodated in a scheme where the smallness of neutrino masses follows from the exchange of superheavy neutral leptons, a la seesaw. Within many grand unified (GUT) models one expects a hierarchy of neutrino masses of the type $m_{\nu_{e}}: m_{\nu_{\mu}}: m_{\nu_{\tau}} \sim m_{u}^{2}: m_{c}^{2}: m_{t}^{2}$. This suggests that if one chooses a $10 \mathrm{eV} \nu_{\tau}$ as the hot dark matter component then one gets $m_{\nu_{\mu}}$ in the range required for the explanation of solar neutrino data. This seesaw scenario also suggests that the forthcoming experiments at CERN $[7,8]$ and the suggested P803 experiment at FNAL [9] may be able to observe $\nu_{\mu}-\nu_{\tau}$ oscillations [6]. However we stress that this numerology relies on many model dependent assumptions about the specific GUT symmetry breaking mechanisms that generate the relevant mass scales.

In this letter we suggest an alternative scenario where radiative corrections associated to new Higgs bosons at the electroweak scale are the origin of the two neutrino mass scales $m_{D M}$ and $m_{\odot}$. We first note that the simplest model of radiative generation of neutrino masses [10], containing just the three usual neutrinos, does not lead naturally to a common explanation of these two scales. In this case, for natural choices of parameters, only one of the neutrinos is ultralight, so there is no MSW effect. Similarly, as in the seesaw mechanism, there is no loop suppression for the ratio $m_{\odot} / m_{D M}$.

We thus consider the simplest extension of the lepton sector which includes a light sterile lepton, $\nu_{S}$. Here one can choose the $\nu_{S}$ mass scale to be $m_{D M}$ [11] or to remain at the ultralight scale $m_{\odot}$, as in the model of ref. [12]. In the latter case it is the $\nu_{\mu}$ and $\nu_{\tau}$ that form the hot dark matter component, while the solar neutrino data are explained by sterile $\nu_{e}$ to $\nu_{S}$ oscillations. In either case no physics is introduced beyond the electroweak scale. For definiteness we focus here on the simplest model where $\nu_{S}$ is at the dark matter scale and the solar neutrino data 
are explained via $\nu_{e}$ to $\nu_{\mu}$ or $\nu_{e}$ to $\nu_{\tau}$ oscillations. We find that in this case, it is possible to reconcile, for reasonable choices of parameters, the existence of a heavy neutrino playing the role of hot dark matter component with the MSW explanation of the solar neutrino data. Apart from the possible $\nu_{e}$ to $\nu_{\tau}$ and $\nu_{\mu}$ to $\nu_{\tau}$ oscillations, our model leads to the possibility of enhanced rates for muon number violating processes $\mu \rightarrow e+\gamma, \mu \rightarrow 3 e$ and $\mu \rightarrow e+J$. Some of these rates can lie within the sensitivities of present experiments. We also discuss the possibility of fitting simultaneously solar, dark matter and atmospheric neutrino data.

\section{Model}

We consider a model based on the $S U(2) \otimes U(1)$ gauge group, which extends the standard model by adding four singlet Higgs bosons $k^{++}, \eta^{+}, h^{+}$and $\sigma$ and one $S U(2) \otimes U(1)$ singlet neutrino $\nu_{S}$. The Yukawa interactions are given by [11]

$$
-\frac{\sqrt{2} m_{i}}{v} \bar{\ell}_{i} \phi e_{R i}+f_{i j} \ell_{i}^{T} C i \tau_{2} \ell_{j} h^{+}+h_{i j} e_{R i}^{T} C e_{R j} k^{++}+\xi_{i} \nu_{S}^{T} C e_{R i} \eta^{+}+\text {h.c. }
$$

where $\ell$ denotes a lepton doublet, $f, h, \xi$ are dimensionless Yukawa couplings. In addition the model contains the following crucial scalar self interactions

$$
\lambda_{k} \sigma h^{-2} k^{++}+\mu_{\eta} \sigma^{*} h^{-} \eta^{+}+h . c .
$$

where $\lambda_{k}$ is dimensionless and $\mu_{\eta}$ has dimensions of mass and its magnitude is at the weak scale.

Defined as above, the model contains a global lepton number symmetry $U(1)_{G}$ assigned canonically to the standard model states. The quantum number assignments and particle content are summarized in Table 1.

This symmetry is broken spontaneously by the vacuum expectation value (VEV) $\langle\sigma\rangle$ thus generating a majoron given by

$$
J=\operatorname{Im} \sigma
$$

The neutrino mass matrix that follows from electroweak and $U(1)_{G}$ violation takes in the basis $\left(\nu_{e}, \nu_{\mu}, \nu_{\tau}, \nu_{S}\right)$ the form

$$
M_{\nu}=\left(\begin{array}{cc}
m_{i j} & M_{i} \\
M_{j} & \mu
\end{array}\right),
$$


Before $U(1)_{G}$ is broken the only nonzero entries are $M_{i}$. In this limit two of the neutrinos are massless and the other two form a Dirac state $\nu_{D M}$ with a mass

$$
m_{\nu_{D M}} \approx \sqrt{M_{e}^{2}+M_{\mu}^{2}+M_{\tau}^{2}}
$$

This mass is induced by the diagram in fig. 1(b). This state is identified by two angles $\theta$ and $\varphi$ defined as

$$
\sin \theta=\frac{M_{e}}{m_{\nu_{D M}}} \quad \tan \varphi=\frac{M_{\mu}}{M_{\tau}}
$$

Here

$$
M_{i}=\frac{\sum_{a} f_{i a} \xi_{a} m_{a}}{32 \pi^{2}} \sin 2 \beta \ln \left(\frac{M_{H_{2}}}{M_{H_{1}}}\right)^{2},
$$

where $\beta$ is the mixing angle of the scalar bosons and $M_{H_{i}}$ are their mass eigenvalues. For suitable choices of Yukawa couplings the Dirac neutrino mass can be at the Dark Matter scale.

The entries $m_{i j}$ and $\mu$ only arise at the 2-loop level from the diagrams in fig. 1(a) and 1(c). They are more highly suppressed also because they involve additional

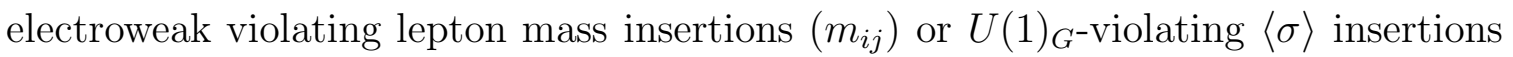
$(\mu)$. They are estimated as

$$
\begin{array}{r}
\mu \approx \frac{\lambda\langle\sigma\rangle \sum_{a, b} h_{a b} \xi_{a} \xi_{b}}{128 \pi^{4}} I_{\mu} \sin ^{2} 2 \beta \\
m_{i j} \approx-\frac{\lambda\langle\sigma\rangle \sum_{a, b} f_{i a} f_{j b} h_{a b} m_{a} m_{b}}{256 \pi^{4} M_{0}^{2}} I_{m},
\end{array}
$$

where $M_{0}$ is a typical Higgs boson mass, $I_{m}$ and $I_{\mu}$ are Feynman integrals typically of order of one $\left(I_{\mu} \rightarrow 0\right.$, when $\left.M_{H_{1}} \rightarrow M_{H_{2}}\right)$. These terms give masses to the lowest-lying neutrinos responsible for the explanation of the solar neutrino data. For suitable values of the parameters, these are in the right range to have a solution of the solar neutrino deficit via matter enhanced transitions. These transitions involve an additional angle needed to diagonalize the resulting light mass matrix relevant for the MSW effect.

The complete form of the charged current weak interaction may be given as

$$
-\frac{g}{\sqrt{2}} W_{\mu}^{-} \sum_{i=1}^{3} \sum_{\alpha=1}^{4} \bar{e}_{i L} \gamma^{\mu} K_{i \alpha} \nu_{\alpha L}+\text { h.c. },
$$

where

$$
K=\left(\begin{array}{llll}
c_{\theta} c_{m} & c_{\theta} s_{m} & s_{\theta} c_{M} & s_{\theta} s_{M} \\
-s_{m} c_{\varphi}-s_{\theta} s_{\varphi} c_{m} & c_{\varphi} c_{m}-s_{\theta} s_{\varphi} s_{m} & s_{\varphi} c_{\theta} c_{M} & s_{\varphi} c_{\theta} s_{M} \\
s_{m} s_{\varphi}-s_{\theta} c_{\varphi} c_{m} & -s_{\varphi} c_{m}-s_{\theta} s_{m} c_{\varphi} & c_{\varphi} c_{\theta} c_{M} & c_{\varphi} c_{\theta} s_{M} \\
0 & 0 & -s_{M} & c_{M}
\end{array}\right)
$$


up to small terms of order $\mathcal{O}\left(m_{M S W} / m_{D M}\right) \lesssim 10^{-4}$. Here the first, second and third rows denote $\nu_{e}, \nu_{\mu}$ and $\nu_{\tau}$ respectively, while the fourth is the sterile neutrino $\nu_{S}$. This matrix $\mathrm{K}$ determines the expected pattern of neutrino oscillations predicted in the model.

\section{Observational Restrictions}

From eq. (11) we can determine all relevant neutrino oscillation probabilities, which are constrained by laboratory and cosmological data. These are given simply as

$$
\begin{aligned}
& P\left(\nu_{e}-\nu_{\tau}\right) \approx \sin ^{2} 2 \theta \cos ^{2} \varphi \sin ^{2} \frac{m_{D M}^{2} L}{2 E} \\
& P\left(\nu_{\mu}-\nu_{\tau}\right) \approx \sin ^{2} 2 \varphi \cos ^{4} \theta \sin ^{2} \frac{m_{D M}^{2} L}{2 E}
\end{aligned}
$$

while the short-scale $\nu_{e}-\nu_{\mu}$ oscillations in vacuo are given as

$$
P\left(\nu_{e}-\nu_{\mu}\right) \approx 2 \sin ^{2} \theta \sin ^{2} \varphi \sin ^{2} \frac{m_{D M}^{2} L}{2 E}
$$

From the existing neutrino-oscillation limits from $\nu_{e}$ disappearance in beam dumps and $\nu_{\mu} \rightarrow \nu_{e}$ we find [13]

$$
\sin ^{2} \theta \lesssim 7 \times 10^{-2}, \quad \sin ^{2} \varphi \lesssim 10^{-3}
$$

where the first limit is from BEBC. These hold for the simple limit case of large $m_{D M}$ where the effects of these oscillations in the laboratory will be averaged out. We have also assumed for simplicity that both $\varphi$ and $\theta$ are small. It follows from the above that the short-scale $\nu_{e}-\nu_{\mu}$ oscillations are highly suppressed since they are proportional to $\sin ^{2} \theta \sin ^{2} \varphi$. Therefore the only important effect $\nu_{e}-\nu_{\mu}$ oscillations is then to explain the solar neutrino deficit via the MSW effect.

The oscillations to $\nu_{S}$ are negligible for laboratory experiments, but they may have important consequences in cosmology. These active-sterile neutrino oscillations can affect primordial nucleosynthesis in two ways. Oscillations that occur before neutrino decoupling can bring the sterile species $\nu_{S}$ into equilibrium at the time when the neutron-to-proton ratio freezes out. On the other hand, oscillations that occur after neutrino decoupling can deplete the $\nu_{e}$ population which also plays an important role in the nucleosynthesis [14].

From eq. (11) one sees that the probabilities for $\nu_{e}-\nu_{s}$ and $\nu_{\mu}-\nu_{s}$ oscillations are proportional to $\sin ^{2} \theta$ and $\sin ^{2} \varphi$, respectively. On the other hand the rate for $\nu_{\tau}-\nu_{s}$ oscillations is maximal, since it is proportional to $\cos ^{2} \theta \cos ^{2} \varphi$. This channel is the 
most efficient one, and it is bounded by the maximum number of neutrino species that can be excited during nucleosynthesis. For a conservative choice $\Delta N_{\nu}<0.8$ these limits are negligible. If however one adopts the limit $\Delta N_{\nu}<0.3$ one obtains $\delta m^{2}<3.4 \times 10^{-6} \mathrm{eV}^{2}[15]$, which would give stringent constraints for the mass matrix. The components $m_{\nu_{\tau} \nu_{\tau}}$ and $m_{\nu_{S} \nu_{S}}$ can be chosen sufficiently small by scaling down the otherwise irrelevant components of the matrix $h$, but also the entries in the $e-\mu$ submatrix contribute to the splitting of the dark matter neutrinos due to mixing. Taking them to be at the MSW scale, and barring the possibility of cancellations, one obtains

$$
\sin ^{2} \varphi \lesssim 6 \times 10^{-6}\left(\frac{10 e V}{m_{D M}}\right), \quad \sin ^{2} \theta \lesssim 1.5 \times 10^{-2}\left(\frac{10 e V}{m_{D M}}\right) .
$$

Both can be satisfied for $\xi_{\tau} \lesssim 10^{-4} \xi_{\mu}$ and $f_{e \mu} \lesssim 0.04 f_{\mu \tau}$. However, these limits can be relaxed somewhat if the majoron coupling is made large $[16,15]$.

There are other cosmological and astrophysical constraints arising from stellar cooling [18] and nucleosynthesis. These constraints involve e.g. sterile neutrino production by $\eta$ exchange, or majoron emission in Compton like scattering. One can verify that they can easily be satisfied, and pose no important constraint for the model since they involve coupling constants $\left(\xi_{e}\right.$, scalar self couplings) that are irrelevant for the neutrino mass matrix.

In this model the heavy neutrino constituting the hot dark matter component in the universe is unstable against majoron emission ${ }^{\S}$. The corresponding lifetime can be estimated as [11]

$$
\tau_{\nu_{D M}} \sim \frac{16 \pi\langle\sigma\rangle^{2}}{m_{D M}\left(r_{1}^{2}+r_{2}^{2}\right)}
$$

where $r_{1} \approx m_{e \tau}+s_{\theta}\left(m_{e e}-m_{\tau \tau}\right)+s_{\varphi} m_{e \mu}$ and $r_{2} \approx m_{\mu \tau}+s_{\varphi}\left(m_{\mu \mu}-m_{\tau \tau}\right)+s_{\theta} m_{e \mu}$. This lifetime can well be longer than the age of the universe, thus justifying our assumption that the heavy neutrino can indeed play the role of the (hot) dark matter component.

The entries of the antisymmetric matrix $f$ are limited by the $e-\mu-\tau$ universality tests. The tree-level amplitudes involving the exchange of a virtual $h$ are proportional to two $f$ vertices. These can affect the ratios $g_{i} / g_{e}(i=\mu, \tau)$ of the electroweak couplings measured in the leptonic $\tau$ decays and in $\mu$ decay. Using the estimates given in [19] we obtain,

$$
\left(\frac{g_{\tau}}{g_{e}}\right)^{2}=1+2 \frac{M_{W}^{2}}{g^{2} M_{h}^{2}}\left(\left|f_{\mu \tau}\right|^{2}-\left|f_{e \mu}\right|^{2}\right),
$$

\footnotetext{
$\S$ This is not an essential feature of our model. In fact we may have explicit breaking of lepton number and in this case no majoron is present and the $\nu_{D M}$ is absolutely stable.
} 


$$
\left(\frac{g_{\mu}}{g_{e}}\right)^{2}=1+2 \frac{M_{W}^{2}}{g^{2} M_{h}^{2}}\left(\left|f_{\mu \tau}\right|^{2}-\left|f_{e \tau}\right|^{2}\right) .
$$

Using simultaneously the determinations [20] $\left(g_{\tau} / g_{e}\right)^{2}=0.952 \pm 0.031,\left(g_{\mu} / g_{e}\right)^{2}=$ $1.016 \pm 0.026$, with correlation 0.40 , we find the $90 \%$ c.l. limits $\left|f_{e \mu}\right|<0.1\left(M_{h} / 100 G e V\right)$, $\left|f_{\mu \tau}\right|<0.06\left(M_{h} / 100 \mathrm{GeV}\right),\left|f_{e \tau}\right|<0.09\left(M_{h} / 100 \mathrm{GeV}\right)$.

The desired mass and mixing pattern can be produced for example choosing $\sin 2 \beta \ln \left(\frac{M_{2}}{M_{1}}\right)^{2} \sim 0.1, \xi_{\mu} \sim 0.05, f_{\mu \tau} \sim 0.02, f_{e \tau} \sim 0.002, h_{\tau \tau} \sim 0.1, \lambda \sim 0.1$, $M_{0} \sim 200 \mathrm{GeV},\langle\sigma\rangle \sim 100 \mathrm{GeV}$ and $\mu_{\eta} \sim 1 \mathrm{GeV}$. This choice is fully consistent with all observational constraints.

To conclude this section we note that another very interesting possibility exists. Suppose that we choose to disregard altogether cosmological nucleosynthesis bounds. In this case our model allows for the intriguing solution to the solar and atmospheric neutrino deficit [17], in addition to the hot dark matter. This is possible if we choose $M_{\mu}$ as the dominant $M_{i}$, corresponding to the choice of $\nu_{\mu}$ and $\nu_{S}$ as the two components of the dark matter neutrino and $\nu_{e}$ to $\nu_{\tau}$ as the channel responsible for the explanation of the solar neutrino data. These maximal mixing oscillations are characterized by a mass difference that can be consistently chosen to lie in the range $10^{-2}-10^{-3} \mathrm{eV}$.

\section{Phenomenology}

The first consequence of our model is the possibility of detectable rates for $\nu_{e}$ to $\nu_{\tau}$ and $\nu_{\mu}$ to $\nu_{\tau}$ oscillations, in the laboratory, eq. (12) and eq. (13). This is possible if we interpret conservatively the BBN limits which restrict the relevant mixing angles, as discussed in the previous section. Our model highlights the ever growing importance of sharpening the nucleosynthesis analysis beyond the present level.

In addition, the interactions present in our model generate several rare processes, which could be observable in the near future. The decay $\mu \rightarrow e \gamma$ proceeds at one-loop level via $h^{+}, \eta^{+}$and $k^{++}$exchange. The typical branching ratio is predicted to be

$$
B(\mu \rightarrow e \gamma) \approx \frac{3 \alpha}{64 \pi} \frac{\left|f_{\tau e} f_{\tau \mu}\right|^{2}}{M_{h}^{4} G_{F}^{2}}
$$

The present $90 \%$ c.l. limit $B(\mu \rightarrow e \gamma)<4.9 \times 10^{-11}$ [21] gives the strongest bound on the product $\left|f_{\tau e} f_{\tau \mu}\right|<0.78 \times 10^{-4}\left(M_{h} / 100 \mathrm{GeV}\right)^{2}$ in our model. Thus with the improved statistics expected at the MEGA experiment proposed at Los Alamos, the decay $\mu \rightarrow e \gamma$ mediated by the scalar boson $h$ could be detected. For instance, for the choice of parameters given above, the resulting branching ratio is $B \sim 10^{-12}$. 
Similarly, the decay $\mu \rightarrow 3 e$ proceeds in our model at the tree level via doubly-charged $k$-exchange. The branching ratio may be estimated as

$$
B(\mu \rightarrow 3 e) \approx \frac{h_{e \mu}^{2} h_{e e}^{2}}{g_{2}^{4}} \frac{M_{W}^{4}}{M_{h}^{4}}
$$

where $g_{2}$ is the $\mathrm{SU}(2)$ gauge coupling. Clearly this branching ratio may be accessible to experiment $[22]$.

Another interesting decay channel is the two-body muon decay with majoron emission, $\mu \rightarrow e J$. This decay is induced at one-loop graph by $h-\eta$ exchange. The relevant coupling may be estimated as

$$
g_{J e \mu} \sim \frac{\langle\sigma\rangle \mu_{\eta}^{2} m_{\mu} f_{e \tau} f_{\mu \tau}}{16 \pi^{2} M_{0}^{4}}
$$

For our sample choice of parameters this is $\sim 10^{-14}$, giving a branching ratio $5 \times 10^{-13}$, well below the observational bound $B R<3 \times 10^{-6}$ [23]. However, for other choices one can obtain much higher values, even larger than the present limit.

On the other hand, the flavour violating tau decays e.g. $\tau \rightarrow e \gamma, \tau \rightarrow \mu \gamma$ $\tau \rightarrow 3 \mu$, etc. may also be present but for reasonable choices they are still far from experimental reach, and similarly for the majoron emitting tau decays.

\section{Conclusions}

We have presented a simple model that reconciles the existence of a hot neutrino dark matter component, as suggested by recent COBE data, with the explanation of the solar neutrino data via the MSW effect. Neutrino masses arise radiatively as a result of new physics in the scalar boson sector at the electroweak scale. The ratio between the solar neutrino mass scale $m_{\odot}$ and the hot dark matter scale $m_{D M}$ is suppressed by quantum mechanical loops.

The presence of a sterile neutrino leads to stringent limits from primordial nucleosynthesis. If only 0.3 extra neutrinos are allowed then we exclude the possibility of observable rates for $\nu_{e} \nu_{\tau}$ or $\nu_{\mu} \nu_{\tau}$ oscillations in the laboratory. However, the uncertainties in the nucleosynthesis limits probably still allow an extra 0.8 light neutrino degrees of freedom at the time when the neutron-to-proton ratio freezes out. Taking this relaxed limit one finds that the oscillation effects could be seen at the new generation of accelerator experiments NOMAD, CHORUS and P803.

In addition the model leads to the possibility of enhanced rates for muon number violating processes $\mu \rightarrow e+\gamma, \mu \rightarrow 3 e$ and $\mu \rightarrow e+J$. These rates can lie within the sensitivities of present and future muon factories. 
Finally, we note that if we choose to ignore BBN limits we can have in this model a common explanation for the atmospheric neutrino deficit via $\nu_{\mu}$ oscillations to a sterile neutrino $\nu_{S}$ with maximal mixing and $10^{-2}-10^{-3} \mathrm{eV}^{2}$.

\section{Acknowledgement}

This work was supported by CICYT and by the Spanish Ministry for Education and Science (D. T. and J. T. P.). J. T. P. also thanks the Magnus Ehrnroth foundation for a grant. We thank A. Joshipura for discussions. 


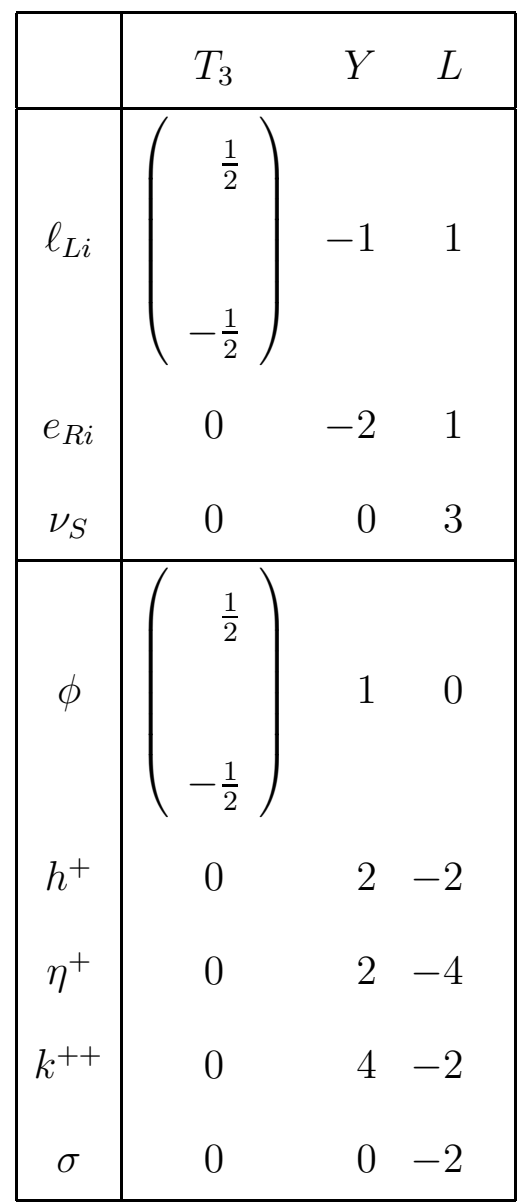

Table 1: $S U(2) \times U(1)_{Y}$ and lepton number assignments of the leptons and Higgs scalars. Quarks are $U(1)_{L}$ singlets. 


\section{References}

[1] G. F. Smoot et al., Berkeley preprint (1992). E. L. Wright et al., Berkeley preprint (1992). R. Shaefer and Q. Shafi, Bartol BA-92-27 (1992). G. Estathiou etal, Oxford OXAST/92/11 (1992). M. Davis etal, Berkeley CfPA-TH-92-016 (1992).

[2] J. R. Davis et al., in Proceedings of the 21th International Cosmic Ray Conference, Vol. 12, ed. R. J. Protheroe (University of Adelaide Press, 1990) p. 293.

[3] A. Abazov et al., Phys. Rev. Lett. 67, 3332 (1991).

[4] P. Anselmann et al., Phys. Lett. B285, 376 (1992).

[5] K. Hirata et al., Phys. Rev. Lett. 65, 1297 (1990); and 66, 9 (1991)

[6] D. K. S. Bludman and P. Langacker, Nucl. Phys. B374, 373 (1992). J. Ellis, J. Lopez and D. V. Nanopoulos, CERN-TH.6569/92.

[7] N. Armenise et al., CERN preprint CERN-SPSC/90-42 (1992).

[8] P. Astier et al., CERN preprint CERN-SPSC/91-21 (1992).

[9] K. Kodama et al., FNAL preprint proposal P803 (1991).

[10] A. Zee, Phys. Lett. B93, 389 (1980).

[11] L. Bento and J. W. F. Valle, Phys. Lett. B264, 373 (1991).

[12] J. T. Peltoniemi, A. Smirnov, and J. W. F. Valle, Phys. Lett. B286, 321 (1992).

[13] Particle Data Group, Phys. Rev. D45, S1 (1992).

[14] R. Barbieri and A. Dolgov, Nucl. Phys. B349, 742 (1991). K. Enqvist, K. Kainulainen, and M. Thomson, Nucl. Phys. B373, 498 (1992).

[15] K. Enqvist, K. Kainulainen, and M. Thomson, Phys. Lett. B 280, 245 (1992).

[16] K. Babu and I. Rothstein, Phys. Lett. B 275, 112 (1992).

[17] K. Hirata etal, Phys. Lett. B 205, 416 (1988). Phys. Lett. B 280, 146 (1992).

[18] J. E. Kim, Phys. Rev. 150, 1 (1987), and references therein.

[19] V. Barger, G. Giudice, and T. Han, Phys. Rev. D 40, 2987 (1989).

[20] E. Nardi, E. Roulet, and D. Tommasini, FERMILAB PUB 91/207-A (1991).

[21] R. Bolton et al., Phys. Rev. Lett. 56, 2461 (1983).

[22] W. Burtl et al., Nucl. Phys. B260, 1 (1985).

[23] A. Jodidio et al., Phys. Rev. D34, 1967 (1986) ; erratum Phys. Rev. D37 (1988) 237. 


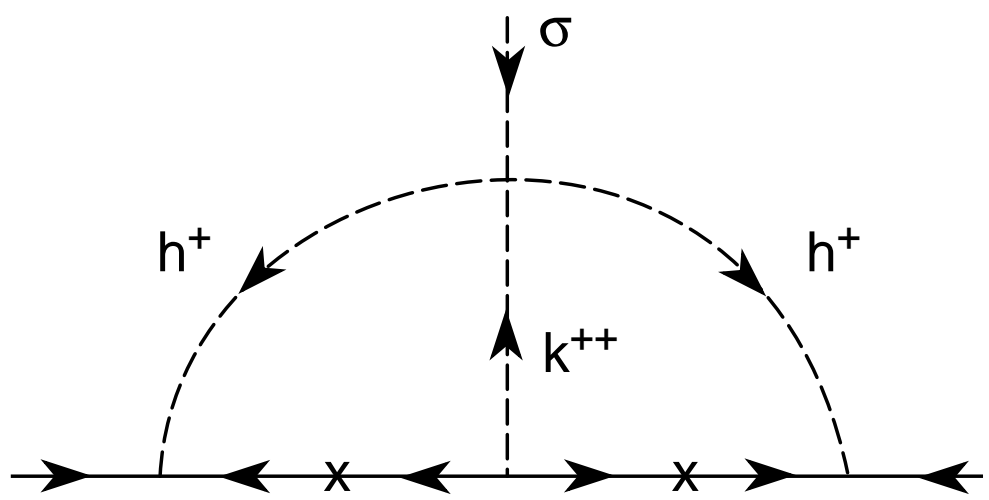

(a)

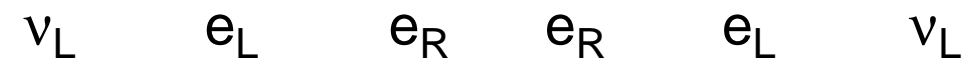

(b)
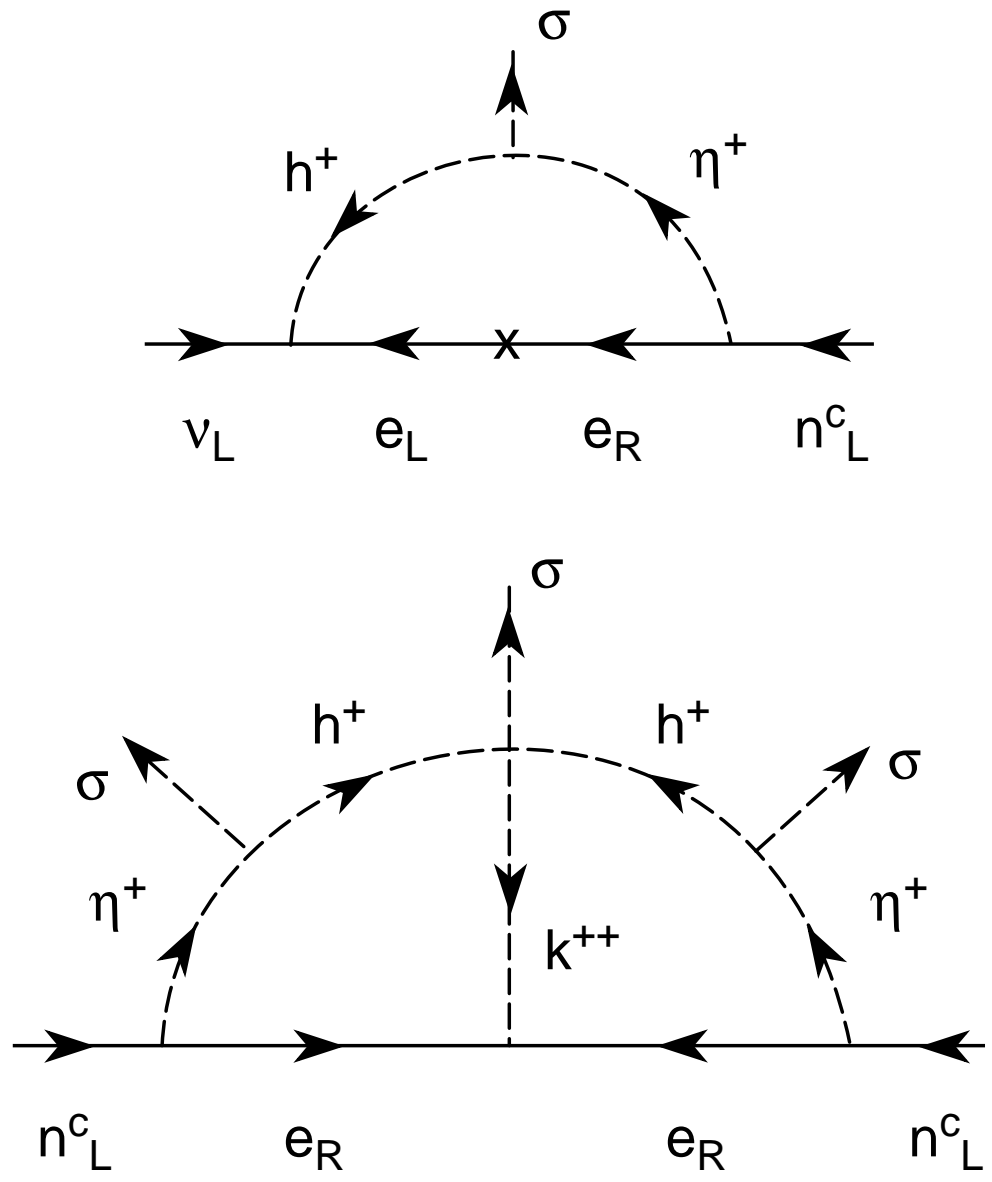

(c)

Fig. 1. Diagrams generating non-zero neutrino masses. (a) and (c) give the small Majorana entries $m_{i j}$ and $\mu$ that can be $\mathrm{O}\left(10^{-3}\right) \mathrm{eV}$ while (b) gives the Dirac mass contribution. 